\title{
Dynamics of Black Holes in Rotating Cores
}

\author{
Jose A. Fiestas ${ }^{1}$ and Rainer Spurzem ${ }^{1}$ \\ ${ }^{1}$ Zentrum für Astronomie, Univ. Heidelberg, Heidelberg, Germany \\ Email: fiestas, spurzem@ari.uni-heidelberg.de
}

Keywords. black hole physics, kinematics and dynamics, galaxies: evolution

We study the dynamical interaction between stars surrounding black holes in self-gravitating galaxy cores by using realistic $N$-body techniques, calibrated by Fokker-Planck approximated models (Fiestas, Spurzem, \& Kim 2006). We study the evolution over the relaxation time (collisional nuclei) of non-spherical (triaxial) models as flattening of the system due to rotation is allowed. We follow the interplay between velocity diffusion due to relaxation and black hole star accretion and study accretion rates and cusp formation in spherically symmetric and axisymmetric models.

Total disruption and accretion of stars occurs inside a disruption radius $r_{\mathrm{d}} \sim r_{\star}\left(M_{\mathrm{BH}} / m_{\star}\right)^{1 / 3}$ of the central $\mathrm{BH}$. The gravitational dominance of the black hole over the surrounding stars vanishes at a radius defined by $r_{\mathrm{h}}=G M_{\mathrm{BH}} / \sigma_{\mathrm{c}}^{2}$. Our initial models are Prugniel-Siemens (Figure 1, left panel) and rotating King profiles (Figure 1, right panel).
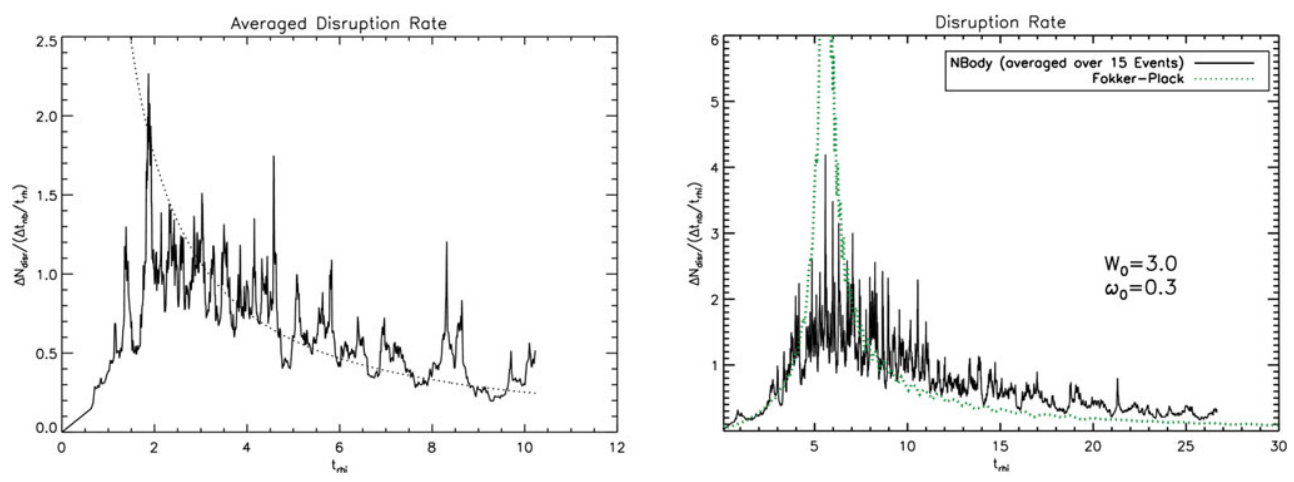

Figure 1. Left: Disruption rates realized in $N$-body models (NBODY6 ++ code) including stellar accretion at the $\mathrm{BH}$ tidal radius. The model follows the theoretical prediction in the post-collapse phase (dashed line), when the Bahcall-Wolf cusp is formed. Right: Disruption rates realized in $N$-body computations (thick lines) and Fokker-Planck rotating (King) models (thin lines) (Fiestas et al. 2010), including stellar accretion at the $\mathrm{BH}$ tidal radius.

\section{References}

Fiestas, J., Spurzem, R., \& Kim, E. 2006, MNRAS, 373, 677

Fiestas, J., \& Spurzem, R. 2010 [arXiv:0810.3800v1] 